A

$\mathrm{B}$

$\mathrm{C}$
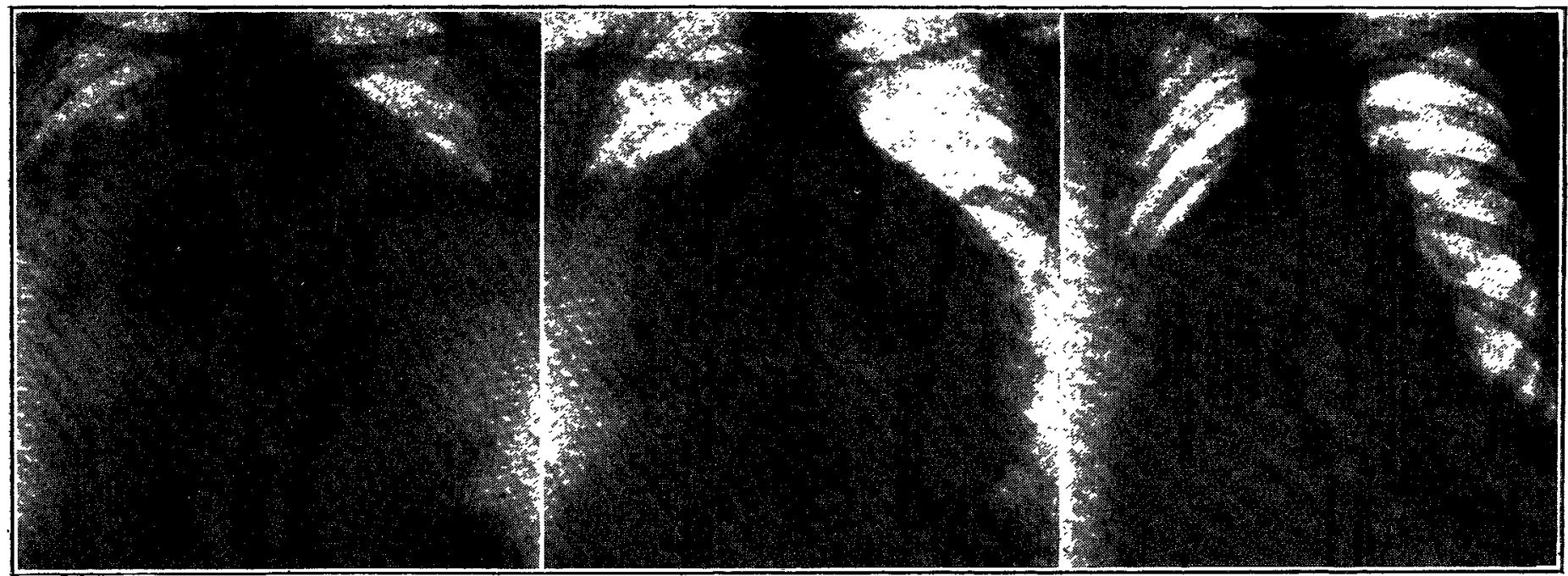

Three X-ray photographs taken from a series which show the gradual decrease of fluid in the pericardium. A, taken on Dec. 27th. Three days later $1000 \mathrm{c.cm}$. of fluid were removed from the pericardial sac. On Jan. $65 \mathrm{~s} 2050 \mathrm{ccm}$. of fluid were removed. B, taken on following day (Feb. 19th).

Further aspirations of the pericardial sac were carried out in conformity with the condition revealed by $X$ ray examination, the patient developing an increasing tolerance, both constitutionally and symptomatically, to the presence of a very large reaccumulation of tluid. On Jan. 6th, 1919, $2050 \mathrm{c.cm}$. were removed. The fluid was darker in colour. No clots. On Jan. 31st over 1000 c.cm. were removed. On Feb. 18th, although the patient exhibited no symptoms of distress, $X$ ray examination revealed a large reaccumulation of fluid-1600 c.cm. were removed. The fluid contained a small number of polynuclear cells, but was sterile.

At the time of the first aspiration there was distinct œdema of the front of the chest wall, but this did not recur to any extent as the pericardial sac refilled. For the first 10 days or so after admission to hospital there was a considerable amount of fever, ranging from $99^{\circ}$ to $103^{\circ}$, but for the last few weeks the temperature chart has shown a subfebrile condition with occasional short rises. In view of the gravity of this case it is remarkable that the pleural and pericardial cavities alone showed any evidence of disease. At the present time (Feb. 25th, 1919) the patient is in fair general health. Although a little dyspnceic there is no orthopncea and the pulse is perfectly regular, as shown by polygraphic tracing.

We consider both the pleural and pericardial inflammation in this patient to be the result of infection of these membranes with tubercle. The cytological picture presented by the predominating lymphocyte count, coupled with the uniform sterility of both pleural and pericardial exudates on ordinary media, clearly point to this conclusion. The special features in this case are the extreme distension of the pericardial sac and the large quantities of fluid removed by aspiration, the marked relief afforded by the early aspirations, and the remarkable tolerance shown in the later stages to the presence of very large reaccumulations of fluid.

\title{
COMMINUTED FRACTURE OF HUMERUS.
}

\section{By WinIFRed F. BUCKLEY, M.R.C.S.}

SURGEON, ENDELL-STREET MILITARY HOSPITAL, LONDON, W.c.

THE following is a case of comminuted fracture of the humerus from the upper end of the shaft to the elbow, which healed and united firmly without the formation of sequestra.

Pte. - - wounded on April 22nd, 1918. The bullet entered the upper surface of the right shoulder and passed longitudinally down the shaft of the humerus; wound of exit over right elbow. He was operated on at the C.C.S. admitted to the Military Hospital, Endell-street, three days later. On admission there was a clean sutured wound on the inner side of the arm about 6 in. long, a small superficial wound the size of a shilling over the head of the humerus, and a wound on the outer side of the elbow-joint leading into the joint. Much swelling and cedema of the

whole arm, forearm, and hand. X ray showed severe comminution of humerus from the elbow to upper end of shaft, with fracture of the olecranon and capitellum of radius. (See figure.)

I treated the fracture first on a broad internal angular splint passing up above the shoulder-joint, and fixed to a counterpoise for eight weeks, then with and aluminium splint ing. There was very slight discharge of pus from the elbowjoint for some weeks. The wounds healed with very Jittle trouble, they were treated with bipp, and the dressings changed about once a fortnight nnder an anæsthetic. The musculo-spiral nerve was completely severed, and was sutured four months after the injury.

The remarkable fact is that the whole shaft united firmly in good line without the formation of $a$ single sequestrum. Probably the bullet passed vertically through the shaft of the humerus, breaking it into small pieses without separating the periosteum. The elbow is anky losed. Power in the fingers and wrist is fairly good, except for the muscles supplied by the musculo-spiral nerve. There is a good movement of the shoulder. The cdema was persistent, and did not subside for three months. The fragments had united firmly in 14 weeks.

Dorset Mental Hospital. - The Dorset County Council has decided to change the name of the county asylum to that of the Dorset Mental Hospital. At present there are 442 male and 602 female patients in the institation. 